This is the peer reviewed version of the following article:

Sex differences in interpretation bias in adolescents, Gluck, R., Lynn, D. A., Dritschel, B. \& Brown, G. R. Mar 2014 In : British Journal of Developmental Psychology. 32, 1, p. 116-122, which has been published in final form at http://dx.doi.org/10.1111/bjdp.12030. This article may be used for noncommercial purposes in accordance With Wiley Terms and Conditions for self-archiving. 


\section{Sex differences in interpretation bias in adolescents}

Rachel L. Gluck ${ }^{1}$, Debra A. Lynn ${ }^{1,2}$, Barbara Dritschel ${ }^{1}$ and Gillian R. Brown ${ }^{1 *}$

${ }^{1}$ School of Psychology \& Neuroscience, University of St Andrews, U.K.

${ }^{2}$ Department of Psychology, University of Stirling, U.K.

Citation: Gluck, R., Lynn, D. A., Dritschel, B. and Brown, G. R. 2014. Sex differences in interpretation bias in adolescents. British Journal of Developmental Psychology 32: 116-122.

*Requests for reprints should be addressed to Dr Gillian R. Brown, School of Psychology and Neuroscience, University of St Andrews, South Street, St Andrews, Fife, KY16 9JP, U.K. (e-mail: grb4@st-andrews.ac.uk) 


\section{Abstract}

Interpretation biases, in which ambiguous information is interpreted negatively, have been hypothesised to place adolescent females at greater risk of developing anxiety and mood disorders than same-aged males. We tested the hypothesis that adolescent girls interpret ambiguous scenarios more negatively, and/or less positively, than sameaged males using the Adolescent Interpretation and Belief Questionnaire ( $N=67,11-15$ years old). We also tested whether adolescent girls and boys differed in judging positive or negative interpretations to be more believable and whether the scenario content (social versus non-social) affected any sex difference in interpretation bias. The results showed that girls had higher average negative interpretation scores than boys, with no sex differences in positive interpretation scores. Girls and boys did not differ on which interpretation they found to be most believable. Both sexes reported that positive interpretations were less likely to come to mind, and were less believable, for social than for non-social scenarios. These results provide preliminary evidence for sex differences in interpretation biases in adolescence and support the hypothesis that social scenarios are a specific source of anxiety to this age group. A greater understanding of the etiology of interpretation biases will potentially enhance sex- and age-specific interventions for anxiety and mood disorders.

Key words: social phobia, gender, interpretive bias, anxiety, puberty, judgement bias 


\section{Introduction}

Women are almost twice as likely to suffer from an anxiety or mood disorder during their lifetimes than are men (Kessler et al., 2005), and, for several disorders, the sex difference in prevalence emerges at adolescence (e.g., depression: Hankin et al., 1998; social phobia: Costello et al., 2003). A negative cognitive style has been hypothesised to be one of several factors that place adolescent females at greater risk than same-aged males of developing clinical anxiety or mood disorders (Hankin and Abramson, 2001; Hyde et al., 2008; Nolen-Hoeksema and Girgus, 1994). However, while adolescent girls are predicted to exhibit stronger negative cognitive biases than same-aged boys in non-clinical samples, relatively few studies have examined this hypothesis (e.g., Bell-Dolan, 1995; van Beek and Debus, 2008). Here, we investigated whether adolescents exhibit sex differences in interpretation bias, defined as the tendency to attribute positive or negative interpretations to ambiguous information (Blanchette and Richards, 2010).

We used the Adolescent Interpretation and Belief Questionnaire (AIBQ; Miers et al., 2008), which presents written scenarios and asks whether specific negative, positive and neutral interpretations come to mind. As the questionnaire presents both positive and negative interpretations, researchers can examine whether some groups of individuals are more negative, and less positive, in their interpretations than other groups, or whether groups differ along just one of these valence dimensions. We predicted that adolescent girls would exhibit higher negative interpretation scores, and/or lower positive interpretation scores, when presented with ambiguous scenarios than same-aged boys. The questionnaire also asks participants to state which of the interpretations they found most believable; thus, we were able to compare whether 
any sex differences in interpretation bias were mirrored by sex differences in belief scores.

As the AIBQ contains an equal number of social and non-social scenarios, we were also able to examine whether sex differences in interpretation and belief scores varied depending upon the content of the scenario. In adulthood, the content of interpretation biases is sometimes specific to a particular sub-disorder; for example, adults with social phobia exhibit negative interpretation biases when evaluating social, but not non-social, information (e.g. Stopa and Clark, 2000). In contrast, relatively little is known about the content specificity of cognitive biases in adolescents (Muris and Field, 2008). We predicted that negative interpretation scores would be higher, and negative interpretations would be more believable, for social than for non-social scenarios, as social issues are a major source of anxiety in this age group (Gullone and King, 1993). We also predicted that girls' responses would be particularly biased towards negative, or away from positive, interpretations for social scenarios, as adolescent girls' self-worth is strongly related to positive social evaluation (e.g., O’Brien and Bierman, 1988).

In summary, we examined whether adolescent girls and boys differ in interpretation bias and belief scores, and whether any sex differences in these biases vary depending upon the scenario content. Given that individuals with anxiety or mood disorders are known to interpret ambiguous scenarios more negatively than other individuals (Blanchette and Richards, 2010; Mathews and MacLeod, 2005), we also needed to test whether sex differences in cognitive biases are instead explained by sex differences in current symptoms of mood disorders. Therefore, we included a measure of depressive symptomology as a co-variate in the analyses of sex differences in interpretation bias scores and belief scores. 


\section{Methods}

\section{Participants and procedure}

The participants were 31 girls (13.6 \pm 0.1 years; range $=12.0-15.2$ years) and 36 boys $(13.4 \pm 0.2$ years; range $=11.3-15.5$ years $)$, recruited from the local population (U.K.) through advertisements in local newspapers and newsletters, and posters at family-oriented venues. The primary language of all participants was English. Ethical clearance was provided by the School of Psychology and Neuroscience Ethics Committee. Each participant was tested individually and completed the Adolescents' Interpretation and Belief Questionnaire, followed by the Revised Child Anxiety and Depression Scale. Receptive vocabulary age of participants was confirmed as being within the normal range for this age group using the British Picture Vocabulary Scale (BPVS; Dunn, Dunn, \& Whetton, 1997). BPVS scores did not differ between the sexes $\left(\right.$ males $=14.8 \pm 0.4$ years, females $=14.5 \pm 0.4$ years: $t$-test, $t_{65}=0.6$, n.s. $)$.

\section{Measures}

Adolescents' Interpretation and Belief Questionnaire (AIBQ; Miers et al., 2008) This questionnaire assesses interpretation bias in adolescents and presents ten scenarios for the participants to imagine themselves in, with half involving social interactions and half involving non-social scenarios. An example of a social scenario is: 'Two classmates, who are standing talking to each other, look at you. Why are they looking at you?' The participant is then provided with three possible responses: one positive ('They like me and want me to go over and join them'), one negative ('They're 
gossiping about me') and one neutral ('They just happen to be looking in my direction'). An example of a non-social scenario is that a person has left his/her bike somewhere and cannot find it on returning to the location, where the possible responses are positive ('I'm looking in the wrong place but it's definitely around here'), negative ('It's been stolen') or neutral ('There are just so many bikes that it's difficult to find it').

For each scenario, the participant rated the anticipated likelihood of each explanation coming to mind if he/she was in that situation (five-point Likert scale with three anchor points; 1 = 'doesn't pop up in my mind', 3 = 'might pop up in my mind', 5 $=$ 'definitely pops up in my mind') and which one of the three explanations the participant found to be the most believable. Neutral explanations were not included in the analyses to allow direct comparison with the study by Miers and colleagues (2008). The mean rating of positive/negative/neutral explanation coming to mind (range $=1-5$ ) and mean belief rating (positive explanation $=1$, neutral explanation $=2$, negative explanation $=3$ ) were calculated separately for the social and non-social scenarios.

Revised Child Anxiety and Depression Scale (RCADS; Chorpita et al., 2000) The RCADS is a self-reported measure of anxiety and depressive symptoms for children and adolescents. The questionnaire has 47 items that require the participant to state how often the item (e.g., 'I get scared when I have to take a test') applies to him/her (four-point Likert scale; 0 = never, 1 = sometimes, 2 = often, 3 = always). The items cover social phobia, major depressive disorder, and a range of anxiety disorders; data from the depression subscale were used as a covariate in our analyses. The scale's internal consistency, reliability and validity are good (Chorpita et al., 2000), and Cronbach's alpha scores are high (Chorpita et al., 2000). 


\section{Results}

\section{$A I B Q$ negative and positive interpretation scores}

A repeated-measures analysis of variance (ANOVA), with valence (negative/positive) and scenario type (social/non-social) as within-subjects variables and sex as a between-subject variable, revealed a significant interaction between valence (negative/positive) and $\operatorname{sex}\left(F_{1,65}=4.36, p=.041\right.$, partial-eta squared $\eta_{p}^{2}=.063$; Figure 1). This significant interaction was due to girls being more likely than boys to bring negative interpretations to mind (simple effects post-hoc tests, $p<.05$ ). Boys were also more likely to bring positive interpretations to mind than negative interpretations $(p<.01)$, while positive interpretation scores did not differ between girls and boys (n.s.). When depressive score was included as a co-variate in the ANOVA, the interaction between sex and valence was reduced to a trend $\left(F_{1,64}=3.10, p=.083\right.$, $\left.\eta_{p}^{2}=.046\right)$.

The interaction between scenario type and sex was not significant $\left(F_{1,65}=.29, \mathrm{p}\right.$ $\left.=.594, \eta_{p}^{2}=.004\right)$, nor was the three-way interaction between valence, scenario type and $\operatorname{sex}\left(F_{1,65}=.21, \mathrm{p}=.651, \eta_{\mathrm{p}}{ }^{2}=.003\right)$. Thus, the sex difference in negative interpretation bias did not depend upon the scenario content. However, there was a significant interaction between scenario type and valence $\left(F_{1,65}=48.30, \mathrm{p}<.001, \mathrm{\eta}_{\mathrm{p}}^{2}=\right.$ .426; Figure 2). Positive interpretations were more likely to come to mind in non-social scenarios than in social scenarios for both sexes $(p<.001)$. Positive interpretations were also more likely to come to mind than negative interpretations in non-social 
scenarios for both sexes $(p<.001)$. No other pairwise comparisons were significant. The interaction between scenario type and valence remained significant when depressive score was included as a co-variate $\left(F_{1,64}=4.91, \mathrm{p}=.030, \mathrm{\eta}_{\mathrm{p}}{ }^{2}=.071\right)$.

\section{AIBQ mean belief rating}

There was no main effect of sex on the belief rating (means and SEMs for females: 0.9 \pm .04 ; males: $0.8 \pm .05 ;$ ANOVA, $\left.F_{1,65}=1.82, p=.182, \eta_{p}^{2}=.027\right)$, and the interaction between scenario type and sex was also non-significant $\left(F_{1,65}=.005, p=.946, \eta_{p}^{2}<\right.$ .001). Thus, despite the sex differences in negative interpretation scores, both girls and boys rated the believability of the interpretations similarly. However, mean belief ratings did differ between scenario types, with belief ratings being significantly higher, i.e. more negative, for social scenario than non-social scenarios (social scenarios: 1.1 \pm .04 ; non-social scenarios: $\left.0.7 \pm .04 ; F_{1,65}=69.41, p<.001, \eta_{p}{ }^{2}=.516\right)$. The main effect of scenario type remained significant when depressive score was included as a co-variate $\left(F_{1,65}=21.25, \mathrm{p}<.001, \mathrm{n}_{\mathrm{p}}^{2}=.249\right)$.

\section{Discussion}

Our results showed that adolescent girls are more likely to bring negative interpretations to mind than same-aged boys when presented with ambiguous scenarios. These data support previous studies that have reported sex differences in negative cognitive bias in adolescents. Adolescent girls have been found to attribute more negative emotions to ambiguous facial expressions (van Beek and Dubas, 2008), attend more to threatening and neutral words (Vasey et al., 1996), and interpret 
peer interactions more negatively than same-aged boys (Bell-Dolan, 1995). Our analyses also showed that including a depressive measure reduced the sex differences in negative interpretation scores, leaving open the possibility that sex differences in negative interpretation are partly explained by current mood symptomology. Despite higher negative interpretation scores across scenarios, adolescent girls did not find negative interpretations to be more believable than did boys, suggesting a dissociation between interpretation of scenarios and judgement of outcome likelihood.

Our results also showed that the sex difference in interpretation bias results from a negative bias in girls, rather than a lack of positive bias. In contrast, Miers and colleagues (2008) reported that adolescent girls are both more negative, and less positive, than same-aged boys. However, Miers and colleagues' (2008) only included individuals with high or average social anxiety scores, potentially leading to the exclusion of low anxiety girls who would be predicted to score high on both negative and positive interpretations. As the sex difference in negative interpretation scores did not vary across scenario type, adolescent girls appear to attribute more negative interpretations to ambiguous scenarios than boys, regardless of content. Thus, the data did not uphold our prediction that girls' responses would be more strongly negative than boys' responses for social, than non-social, scenarios.

Interpretation biases differed according to the content of the ambiguous information across both sexes, with social scenarios being interpreted less positively than non-social scenarios. Adolescents also found negative interpretations to be more believable for social than for non-social scenarios. The finding that both interpretation and belief scores exhibit content specificity in an adolescent population is consistent with other evidence that social issues, such as peer evaluation, are a key source of 
concern in this age group (Westenberg et al., 2007). Future studies could investigate whether sex differences in interpretation bias are also present in younger populations, in order to shed light on whether sex differences in interpretation bias precede the emergence of sex differences in anxiety and mood disorders. If interpretation biases are found to play a role in enhancing the vulnerability of adolescents to anxiety and mood disorders, a greater understanding the etiology of such biases, including the emergence of content specificity, could improve sex- and age-specific interventions and treatments (Rapee et al., 2009; Sauter et al., 2009).

\section{Acknowledgements}

We are particularly grateful to an anonymous reviewer for many valuable comments on the manuscript and to Anne Miers for providing the AIBQ.

\section{References}

Bell-Dolan, D. J. (1995). Social cue interpretations of anxious children. Journal of Clinical Child Psychology, 24, 1-10. doi:10.1207/s15374424jccp2401_1

Blanchette, I., \& Richards, A. (2010). The influence of affect on higher level cognition: a review of research on interpretation, judgement, decision making and reasoning. Cognition and Emotion, 24, 561-595. doi:10.1080/02699930903132496

Chorpita, B. F., Yim, L., Moffitt, C., Umemoto, L. A. \& Francis, S. E. (2000). Assessment of symptoms of DSM-IV anxiety and depression in children: a revised child anxiety and depression scale. Behaviour Research and Therapy, 38, 835-855. doi:10.1016/S0005-7967(99)00130-8 
Costello, E.J., Mustillo, S., Erkanli, A., Keeler, G., \& Angold, A. (2003). Prevalence and development of psychiatric disorders in childhood and adolescence. Archives of General Psychiatry, 60, 837-344. doi:10.1001/archpsyc.60.8.837

Dunn, L. M., Dunn, L. M., \& Whetton, C. (1997). The British Picture Vocabulary Scale. NFER-Nelson, Windsor.

Gullone, E, \& King, N. J. (1993). The fears of youth in the 1990s: contemporary normative data. Journal of Genetic Psychology, 154, 137-153. doi:10.1080/00221325.1993.9914728

Hankin, B. L., \& Abramson, L. Y. (2001). Development of gender differences in depression: an elaborated cognitive vulnerability-transactional stress theory. Psychological Bulletin, 127, 773-796. doi:10.1037/0033-2909.127.6.773

Hankin, B. L., Abramson, L. Y., Moffitt, T. E., Silva, P. A., McGee, R., \& Angell, K. E. (1998). Development of depression from preadolescence to young adulthood: emerging gender differences in a 10-year longitudinal study. Journal of Abnormal Psychology, 107, 128-140. doi:10.1037/0021-843X.107.1.128

Hyde, J. S., Mezulis, A. H., \& Abramson, L. Y. (2008). The ABCs of depression: integrating affective, biological, and cognitive models to explain the emergence of gender differences in depression. Psychological Review, 115, 291-313. doi: 10.1037/0033-295X.115.2.291

Kessler, R. C., Berglund, P., Demler, O., Jin, R., Merikangas, K. R., \& Walters, E. E. (2005). Lifetime prevalence and age-of-onset contributions of DSM-IV in the National Comorbidity Survey Replication. Archives of General Psychiatry, 62, 593-602. doi:10.1001/archpsyc.62.6.593

Mathews, A., \& MacLeod, C. (2005). Cognitive vulnerability to emotional disorders. Annual Review of Clinical Psychology, 1, 167-195. doi:10.1146/annurev.clinpsy.1.102803.143916

Miers, A. C., Blöte, A. W., Bögels, S. M., \& Westenberg, P. M. (2008). Interpretation bias and social anxiety in adolescents. Journal of Anxiety Disorders, 22, 14621471. doi:10.1016/j.janxdis.2008.02.010 
Muris, P., \& Field. A. P. (2008). Distorted cognition and pathological anxiety in children and adolescents. Cognition and Emotion, 22, 395-421. doi:10.1080/02699930701843450

Nolen-Hoeksema, S., \& Girgus, J. S. (1994). The emergence of gender differences in depression during adolescence. Psychological Bulletin, 115, 424-443. doi:10.1037/0033-2909.115.3.424

O'Brien, S. F., \& Bierman, K. L. (1988). Conceptions and perceived influence of peer groups: interviews with preadolescents and adolescents. Child Development, 59, 1360-1365. doi:10.1111/j.1467-8624.1988.tb01504.x

Rapee, R. M., Schniering, C. A., \& Hudson, J. L. (2009). Anxiety disorders during childhood and adolescence: origins and treatment. Annual Review of Clinical Psychology, 5, 311-341. doi:10.1146/annurev.clinpsy.032408.153628

Sauter, F. M., Heyne, D., \& Westenberg, P. M. (2009). Cognitive behavior therapy for anxious adolescents: developmental influences on treatment design and delivery. Clinical Child and Family Psychology Review, 12, 310-335. doi:10.1007/s10567-009-0058-z

Stopa, L., \& Clark, D. M. (2000). Social phobia and interpretation of social events. Behaviour Research and Therapy, 38, 273-283. doi:10.1016/S00057967(99)00043-1

van Beek, Y., \& Debus, J. S. (2008). Age and gender differences in decoding basic and non-basic facial expressions in late childhood and early adolescence. Journal of Nonverbal Behavior, 32, 37-52. doi:10.1007/s10919-007-0040-8

Vasey, M. W., El-Hag, N., \& Daleiden, E. L. (1996). Anxiety and the processing of emotionally threatening stimuli: distinctive patterns of selective attention among high- and low-test-anxious children. Child Development, 67, 1173-1185. doi:10.1111/j.1467-8624.1996.tb01789.x

Westenberg, P. M., Gullone, E., Bokhorst, C. L., Heyne, D. A., \& King, N. J. (2007). Social evaluation fear in childhood and adolescence: normative developmental course and continuity of individual differences. British Journal of Developmental Psychology, 25, 471-483. doi:10.1348/026151006X173099 


\section{Figure legends}

Figure 1 Mean rating for negative and positive explanations coming to mind for boys and girls in the AIBQ (means \pm SEMs). * indicates $p \leq .05$ in post-hoc tests.

Figure 2 Mean rating of negative and positive explanations coming to mind in the social and non-social scenarios of the AIBQ (means \pm SEMs). ${ }^{* *}$ indicates $p \leq .001$ in post-hoc tests. 
Figure 1

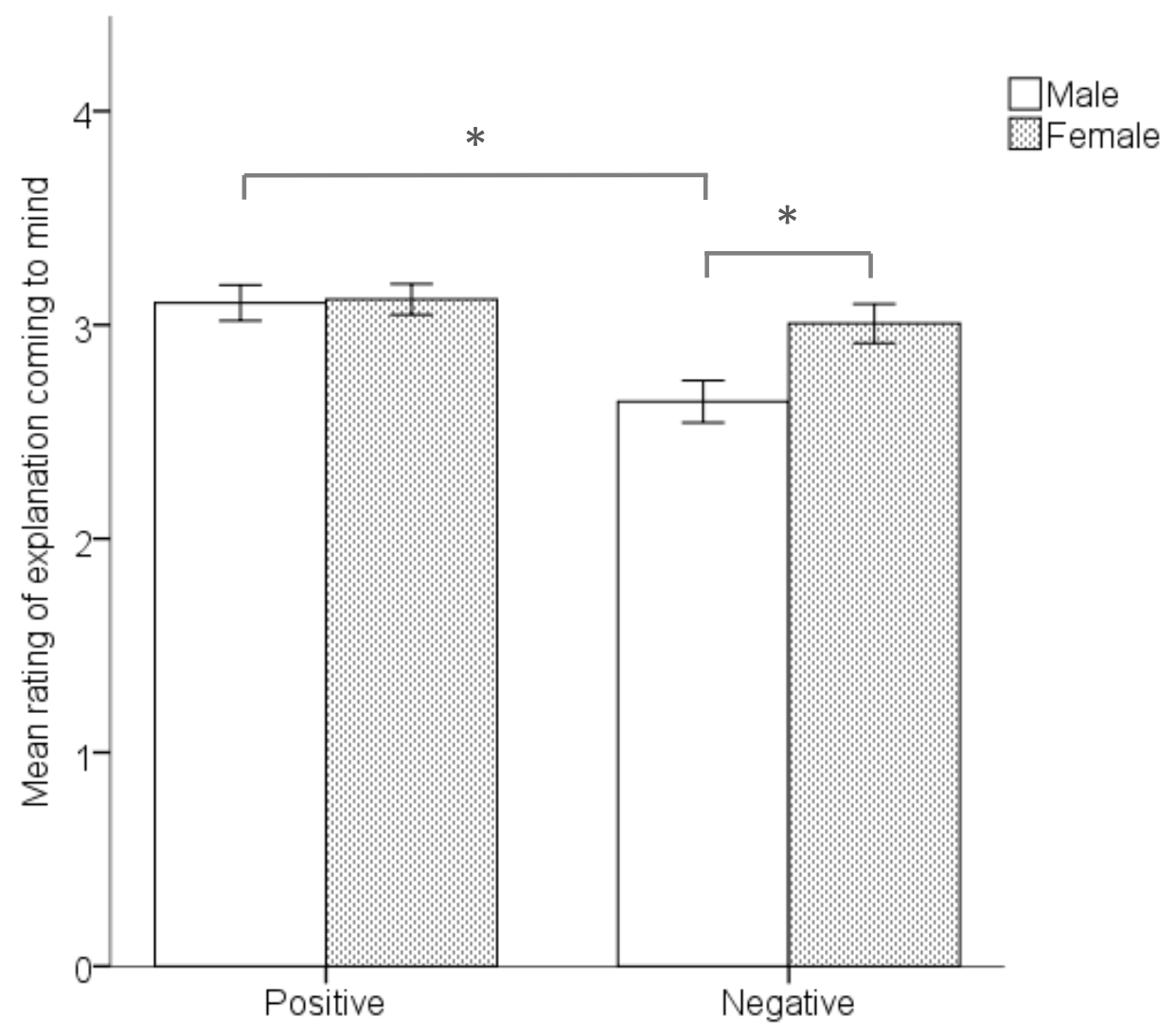


Figure 2

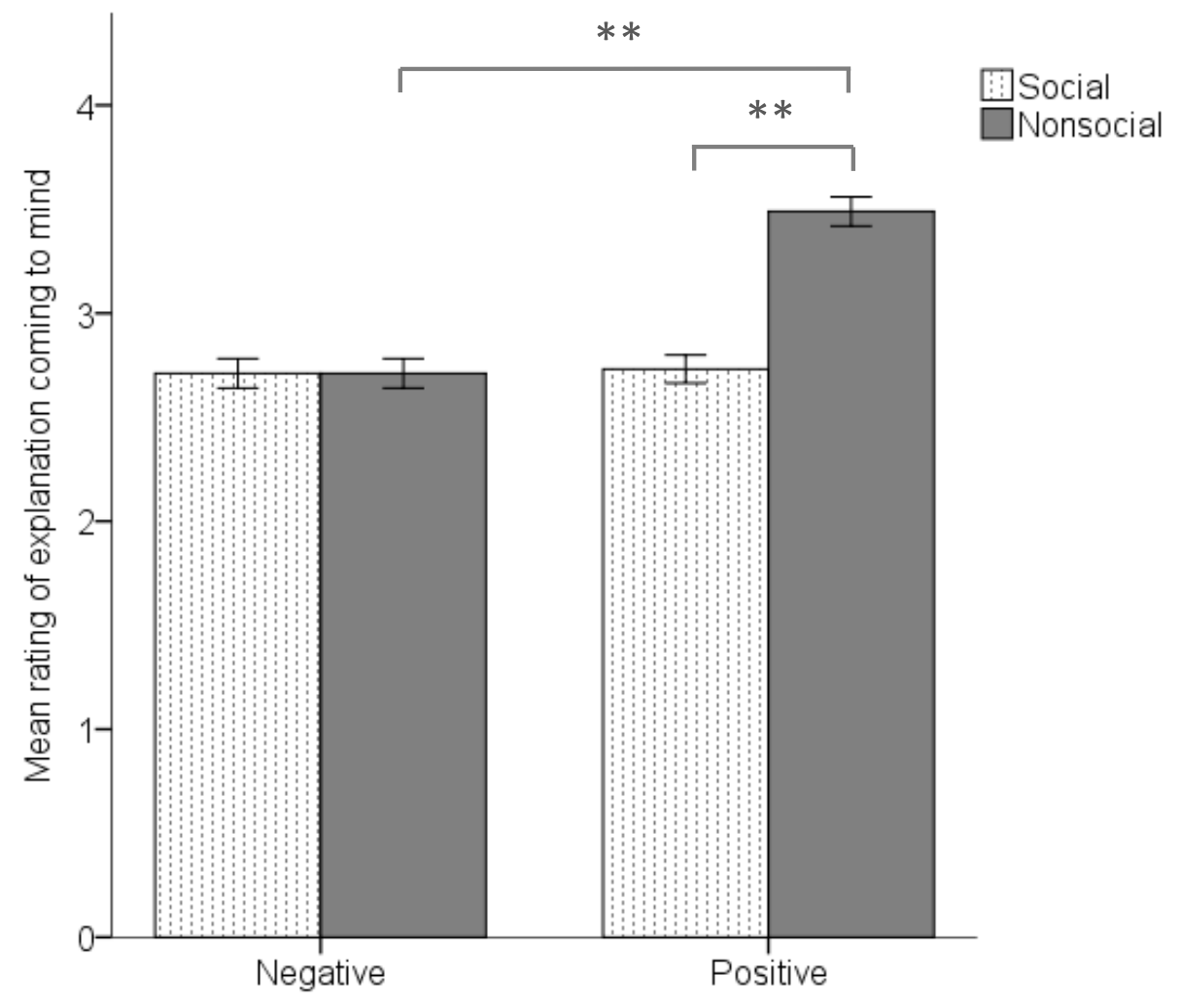

